\title{
Tensile impact behaviour of 3D printed parts on FFF/FDM printer Zortrax M200
}

\author{
Ales Mizera ${ }^{1, *}$, Martin Bednarik ${ }^{1}$, Martin Mizera $^{1}$, Katarina $_{\text {Tomanova }}^{2}$, and Martin Mohorko ${ }^{3}$ \\ ${ }^{1}$ Tomas Bata University in Zlin, nam. T. G. Masaryka 5555, 76001 Zlin, Czech Republic \\ ${ }^{2}$ Slovak University of Technology in Bratislava, Department of Polymer Processing, Vazovova 5, 81243 Bratislava, Slovakia \\ ${ }^{3}$ Siemens, s.r.o. branch of electromotors Frenstat, Markova 952, 74401 Frenstat pod Radhostem, Czech Republic
}

\begin{abstract}
To obtain the deeper knowledge about the mechanical behaviour of 3D printed polymeric materials it is necessary to study the material properties from the beginning to the end. The commonly processed polymeric materials (via injection moulding etc.) are already deeply studied and evaluated, but $3 \mathrm{D}$ printed specimens in the various orientation build are not yet. In this study the tensile impact test specimens were fabricated via a desktop material extrusion 3D printer Zortrax M200 processing ABS and HIPS in build orientation XY. The 3D printed tensile impact test specimens were examined to compare the effect of layer thickness. Impact pendulum Zwick HIT50P was used for tensile impact tests according to ISO 8256 standard. Optical microscopy was utilized to perform fractography on impact test specimens to explore the effect of the layer thickness on the fracture surface morphology of the failed specimens. This study demonstrates the need for material testing for specific processing as additive manufacturing technologies.
\end{abstract}

\section{Introduction}

Fused deposition modelling (FDM) is a manufacturing process using which a prototype or model is produced. FDM was invented by Crump in later 1980s and was commercialized since 1990. Another nomenclature of this process is fused filament fabrication (FFF) or plastic jet printing (PJP). The transportation of the material is supplied using the head of 3D printer with a Teflon tube guide. The polymeric material for $3 \mathrm{D}$ printing is in the shape of the wire. The principle of FDM process is to create the workpiece layer by layer in the z-axes. The head of the extruder with the nozzle is shifted in the $\mathrm{x}$ axis and the direction of y-axes an electric motor, metal rails and belt. When the process starts, after applying of the first layer, the work platform is lowered to a height of already applied layer in the direction of z-axis and the applying of other layer is started until the prototype is finished [1].

Nowadays, 3D printing is used in many branches. From prototype production, automotive, aerospace and defence industries to the health-care where medical products such as patient-specific guides, 3D-printed prosthetics and 3D-printed anatomical models, 3Dprinted hearing aids etc. are produced [2].

One team of the scientists used acrylonitrilebutadiene-styrene (ABS) and polylactic acid (PLA) to the production of the scaffold for cartilage and nucleus pulposus tissue regeneration. The scaffolds had the same construction, porosity and the average weight for both of materials. After measurement of stiffness immediately after printing and 21 days after printing, the stiffness was almost the same, non-changed in time, but different for both materials. The Young's Modulus of both materials was also almost non-changed in time - ABS around 200 $\mathrm{kPa}$ and PLA around $500 \mathrm{kPa}$ [3].

To produce the product of the best quality, the print orientation and the layer thickness are important for the improvement of the bonding strength of FDM products. From the adhesion point of view, the edgewise orientation had the highest adhesion strength in lower layer thicknesses. The flat orientation reached the highest adhesion strength in higher layer thicknesses [4]. FDM technology was also used for the production of two types of scaffold for the bone tissue. The scaffolds were created from PLA and were of the defined inner structure and defined shape. After 3D printing, they were seeded by osteosarcoma cells. It was found that proliferation was satisfying for both types of the scaffold and the porosity of the samples was $30 \%$ and $50 \%$, which confirmed the new finding that it is not necessary to create the porosity of $90 \%$ as is recommended. The size of pore about $0.7 \mathrm{~mm}$ showed that it is not necessary to keep the pore size $0.2-0.35 \mathrm{~mm}$ as is recommended [5]. Other investigations are also concentrated on 3D print in the medicine $[6,7]$.

Keles, Blevins and Bowman investigated 3D printed specimens from ABS plus-P430 in three different build orientation and these specimens were subjected to the tensile test. It was found that the $\mathrm{xz}$ orientation for specimens resulted in the highest average fracture strength for the specimens with and without the hole [8].

Corresponding author: mizera@,utb.cz 
Table 1. 3D printed part maximum force statistical evaluation.

\begin{tabular}{|l|l|c|c|c|c|c|}
\hline \multirow{2}{*}{ Maximum force [N] } & Material & \multicolumn{3}{|c|}{ Z-ABS } & \multicolumn{2}{c|}{ Z-HIPS } \\
\cline { 2 - 7 } & Layer [mm] & $\mathbf{0 . 0 9}$ & $\mathbf{0 . 1 9}$ & $\mathbf{0 . 2 9}$ & $\mathbf{0 . 1 9}$ & $\mathbf{0 . 2 9}$ \\
\hline Number of measurements & 15 & 15 & 15 & 15 & 15 \\
\hline Arithmetic mean & 525 & 510 & 441 & 338 & 276 \\
\hline Standard deviation & 76 & 57 & 62 & 20 & 22 \\
\hline Minimum value & 426 & 403 & 372 & 303 & 235 \\
\hline Median & 493 & 522 & 424 & 347 & 279 \\
\hline Maximum value & 670 & 594 & 581 & 357 & 305 \\
\hline Variation coefficient & 14 & 11 & 14 & 6 & 8 \\
\hline
\end{tabular}

Table 2. All consumed impact work statistical evaluation.

\begin{tabular}{|l|l|c|c|c|c|c|}
\hline \multirow{2}{*}{ All consumed impact work $[\mathbf{J}]$} & Material & \multicolumn{3}{|c|}{ Z-ABS } & \multicolumn{3}{c|}{ Z-HIPS } \\
\cline { 2 - 7 } & Layer $[\mathbf{m m}]$ & $\mathbf{0 . 0 9}$ & $\mathbf{0 . 1 9}$ & $\mathbf{0 . 2 9}$ & $\mathbf{0 . 1 9}$ & $\mathbf{0 . 2 9}$ \\
\hline Number of measurements & 15 & 15 & 15 & 15 & 15 \\
\hline Arithmetic mean & 0.55 & 0.55 & 0.57 & 0.43 & 0.41 \\
\hline Standard deviation & 0.04 & 0.03 & 0.04 & 0.02 & 0.01 \\
\hline Minimum value & 0.50 & 0.49 & 0.51 & 0.39 & 0.38 \\
\hline Median & 0.55 & 0.55 & 0.56 & 0.44 & 0.42 \\
\hline Maximum value & 0.62 & 0.61 & 0.64 & 0.48 & 0.43 \\
\hline Variation coefficient & 7.14 & 5.65 & 6.87 & 5.79 & 3.43 \\
\hline
\end{tabular}

Milde, Morovič and Blaha used in their experiment the same $3 \mathrm{D}$ print as in our case. In their case they printed the upper teeth model with the different layer thickness. They found out that the lowest shrinkage effect of the printed upper teeth model was found on the parts, where the amount of the single layers was the highest [9].

Other study is concentrated on the tensile behaviour of 3D printed specimens. In this study the Young's modulus differs in all series which was caused by the air gaps in the inner structure of the specimens [10].

Our goal in this study is to compare tensile impact behaviour of polymers ABS and high impact polystyrene used for 3D print using printer Zortrax M200.

\section{Experimental}

For production of the tested samples a desktop grade FFF/FDM (Fused Filament Fabrication/Fused Deposition Modeling) 3D printer Zortrax M200 was fed with ABS and HIPS filaments with a specified diameter of $1.75 \mathrm{~mm}$ supplied by Zortrax company to fabricate specimens for tensile-impact testing type T2 according to the dimensions described in the ISO 8256 standard. Sample sets $(n=15)$ were printed in XY orientation. The G-code was made by software Z-Suite v1.12.2 which is part of the $3 \mathrm{D}$ printer pack. Printing process parameters are set as high quality and maximum infill, other process parameters are set automatically by software as an optimal value which company Zortrax recommended for each filament separately. Three heights of layers 0.09 ,
0.19 and 0.29 were used. For HIPS just two heights of layers 0.19 and 0.29 was used. Measurement of layer thickness was measured by optical microscope Zeiss Axio Scope A1 and subsequently photographed for easier evaluation of the results.

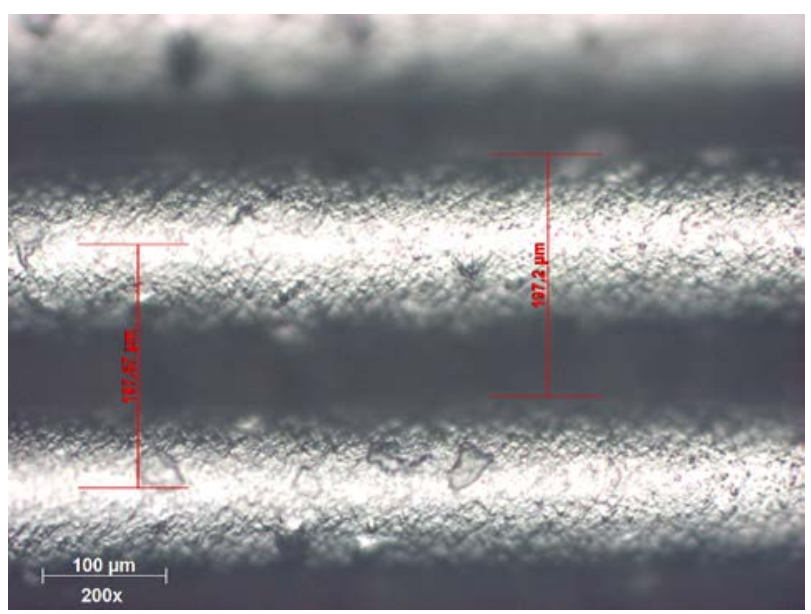

Fig. 1. ABS layer measurement for printed layer $0.19 \mathrm{~mm}$.

Before own testing, the test samples were conditioned for 168 hours at temperature $23{ }^{\circ} \mathrm{C}$ and relative humidity $50 \%$. 3D printed pars were tested on tensile impact test machine Zwick HIT50P according to ISO 8256 standard in the air at ambient temperature (23 ${ }^{\circ} \mathrm{C}$ ). In this test the impact pendulum with potential energy $15 \mathrm{~J}$ was used. 15 samples were tested and values of maximum impact force, all consumed impact work and impact strength were evaluated in programs 
TestXpert II, MiniTab 16 and MS Excel 2016. After the impact test the fracture surface was photographed, discussed and evaluated.

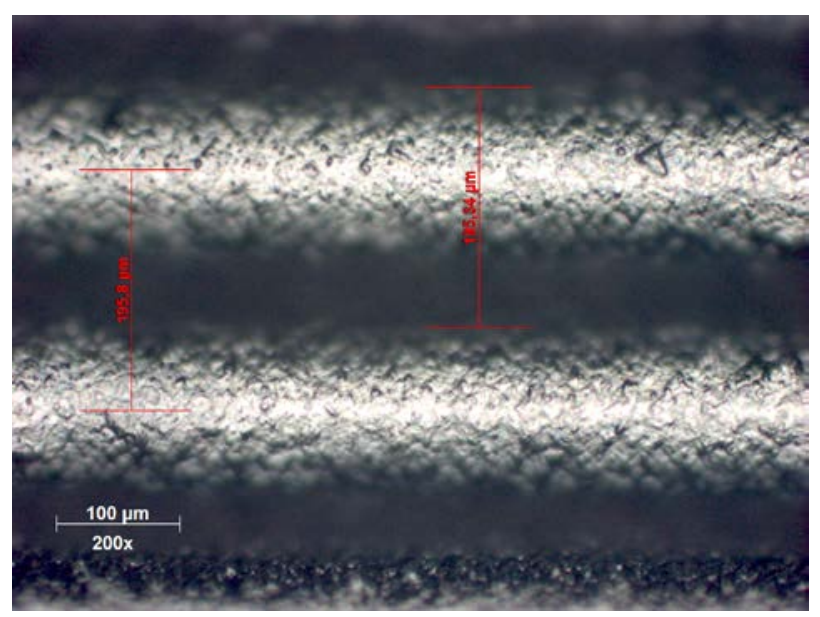

Fig. 2. HIPS layer measurement for printed layer $0.19 \mathrm{~mm}$.

\section{Results and discussion}

In Figure 1 and 2 are depicted two layers at $0.19 \mathrm{~mm}$. From optical measurement on microscope Zeiss Axio Scope A1 was found the value of layer thickness 0.1977 and $0.1958 \mathrm{~mm}$ for ABS and HIPS, respectively. As can be seen, the surface layers are almost equal, which shows the good accuracy of the 3D print jet.

\subsection{Maximum impact force}

Statistical evaluation of maximum impact force is shown in Table 1. From this table can be observed that increasing the thickness of layer causes decreasing of maximum impact force. Graphical comparison of maximum impact force at the different layer is depicted in Figure 3. In this figure median as a main statistical parameter is used. The ABS value of maximum impact force at layer 0.09 and $0.19 \mathrm{~mm}$ is almost similar then is rapidly decreasing at layer $0.29 \mathrm{~mm}$. The same tendency is observed for HIPS at 0.19 and $0.29 \mathrm{~mm}$. The samples with $0.09 \mathrm{~mm}$ layer thickness were not printed, it is not available on Zortrax M200.

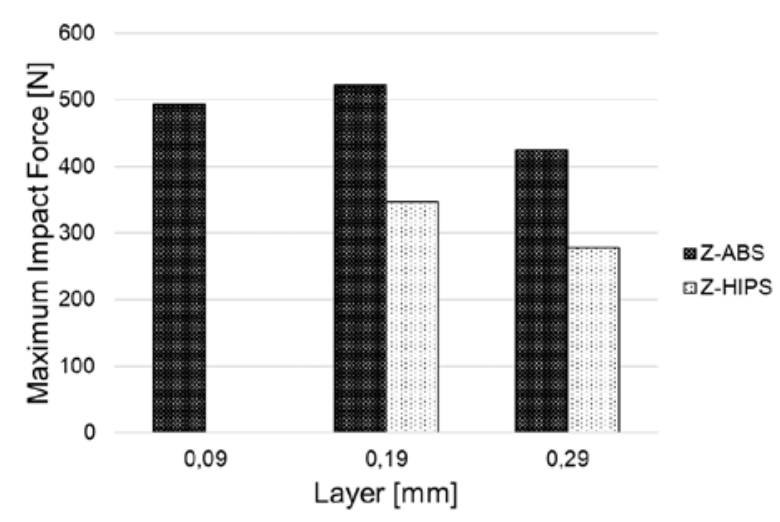

Fig. 3. Comparison of maximum impact force median.

\subsection{All consumed impact work}

Statistical evaluation of all consumed impact work is shown in Table 2. From this table can be seen that the thickness of layer does not cause change of all consumed impact work for ABS. The value is laying in error bars. On the other hand, HIPS value at thickness layer 0.19 $\mathrm{mm}$ is decreasing about of $0.02 \mathrm{~J}$ in comparison with printed layer $0.29 \mathrm{~mm}$. Graphical comparison of all consumed impact work at the different layer is depicted in Figure 4. In this figure median as a main statistical parameter is used. There is shown decrease of all consumed impact work from 0.55 (ABS) to $0.43 \mathrm{~J}$ (HIPS) at the thickness layer $0.19 \mathrm{~mm}$.

\subsection{Impact strength}

Statistical evaluation of impact strength is visible in Table 3. The impact strength is depending on impact work and the cross-section area of fracture surface. This area is changing on the layer thickness. From Figure 5 can be seen that impact strength is decreasing with increasing thickness layer for both used materials. The decreasing is about of $15 \%$ (between layer 0.09 and 0.29 ) for $\mathrm{ABS}$ and $30 \%$ (between layer 0.19 and 0.29 ) for HIPS.

Table 3. 3D printed part impact strength statistical evaluation.

\begin{tabular}{|l|l|c|c|c|c|c|}
\hline \multirow{2}{*}{ Impact strength $\left[\mathbf{k J} / \mathbf{m}^{2}\right]$} & Material & \multicolumn{3}{|c|}{ Z-ABS } & \multicolumn{2}{c|}{ Z-HIPS } \\
\cline { 2 - 7 } & Layer $[\mathbf{m m}]$ & $\mathbf{0 . 0 9}$ & $\mathbf{0 . 1 9}$ & $\mathbf{0 . 2 9}$ & $\mathbf{0 . 1 9}$ & $\mathbf{0 . 2 9}$ \\
\hline Number of measurements & 15 & 15 & 15 & 15 & 15 \\
\hline Arithmetic mean & 32.7 & 31.7 & 28.3 & 18.6 & 12.9 \\
\hline Standard deviation & 2.9 & 1.7 & 2.5 & 1.0 & 0.6 \\
\hline Minimum value & 28.6 & 29.4 & 24.6 & 16.9 & 12.1 \\
\hline Median & 32.6 & 31.3 & 27.9 & 18.5 & 12.8 \\
\hline Maximum value & 37.6 & 34.1 & 32.6 & 20.2 & 14.1 \\
\hline Variation coefficient & 8.7 & 5.4 & 8.7 & 5.5 & 4.8 \\
\hline
\end{tabular}




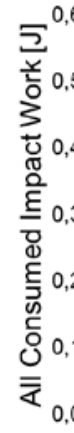
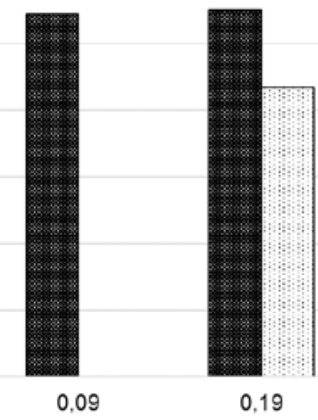

Layer $[\mathrm{mm}]$

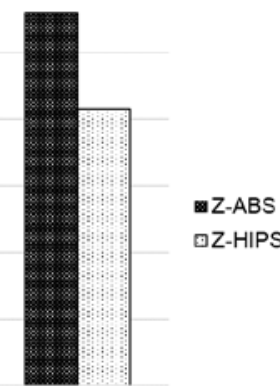

Fig. 4. Comparison of all consumed impact work median.

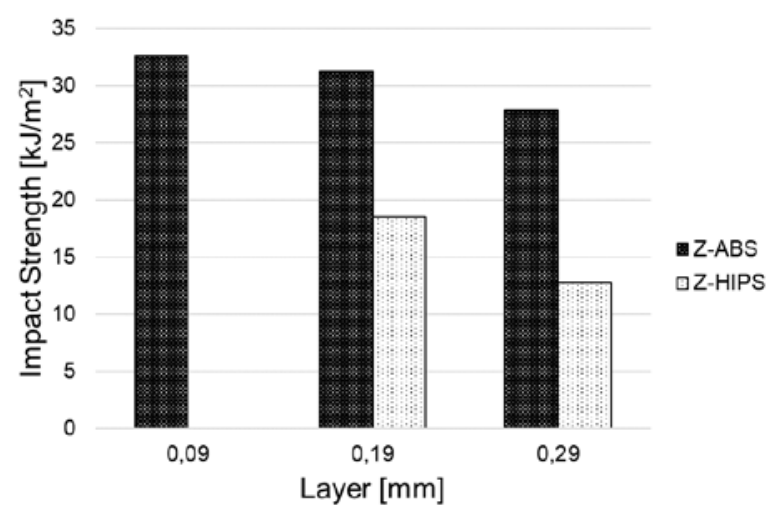

Fig. 5. Comparison of impact strength median.

\subsection{Fracture surface evaluation}

In Figures 6, 7 and 8 are depicted fracture surfaces of ABS specimens after the test. It can be seen from these figures that the layer 0.09 is more compact. With increasing layer height, the layers are separated. For layer 0.29 , the contact area is several times smaller than the lower layers, resulting in a deterioration in the measured properties.

In Figures 9 and 10 are depicted fracture surfaces of HIPS specimens after the tensile impact test. There is observed the same tendency as for ABS material.

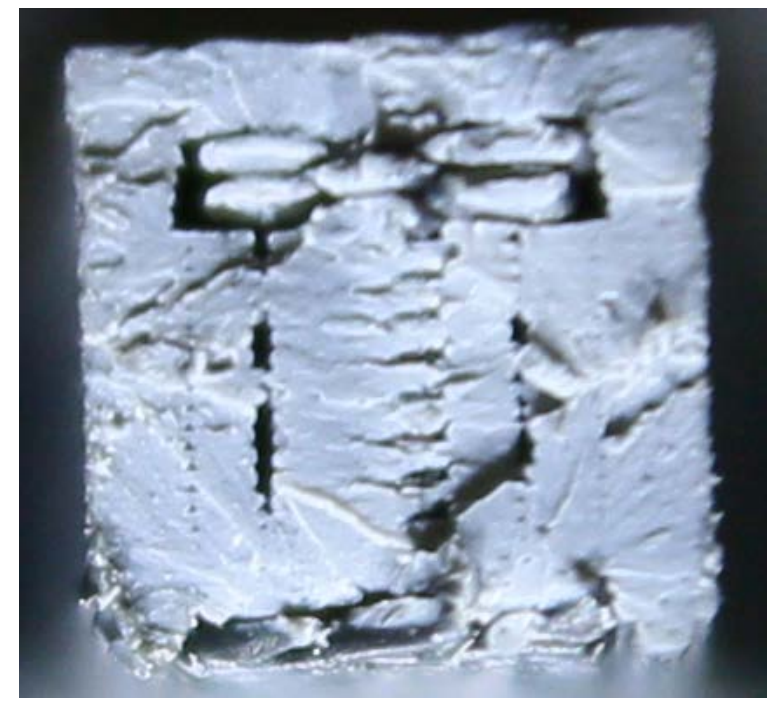

Fig. 6. ABS fracture surface at $0.09 \mathrm{~mm}$ printed layer.

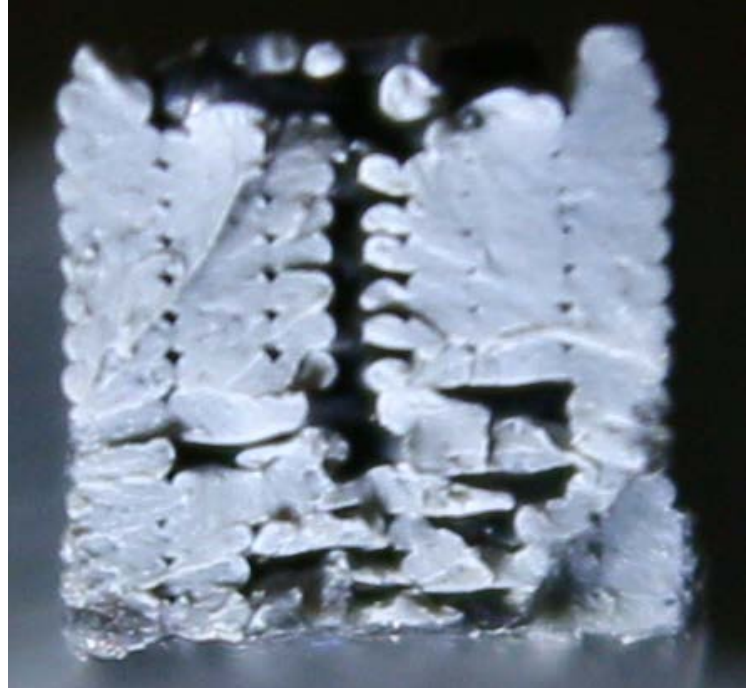

Fig. 7. ABS fracture surface at $0.19 \mathrm{~mm}$ printed layer.

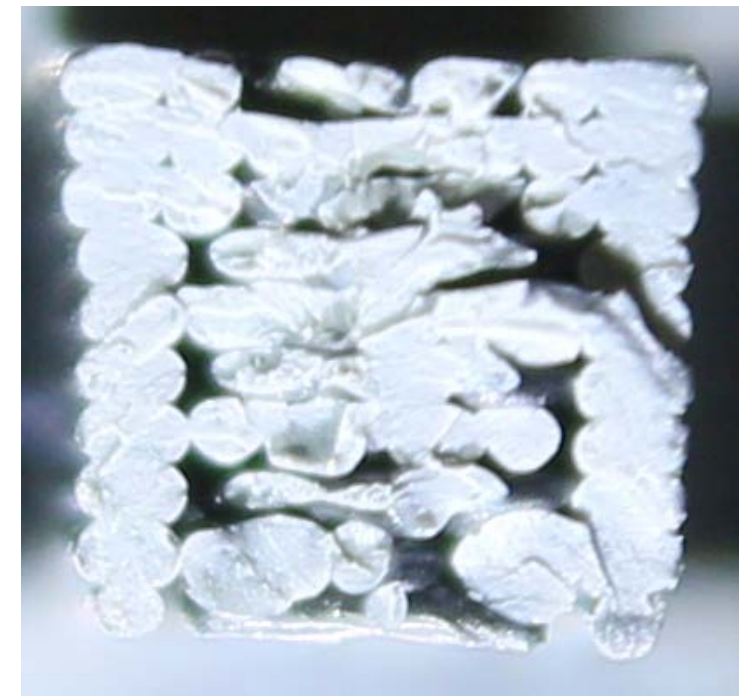

Fig. 8. ABS fracture surface at $0.29 \mathrm{~mm}$ printed layer.

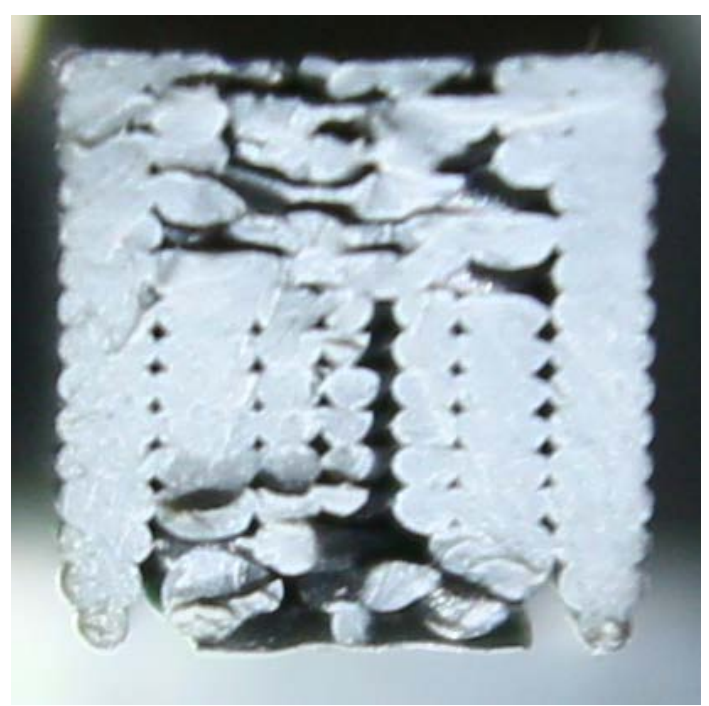

Fig. 9. HIPS fracture surface at $0.19 \mathrm{~mm}$ printed layer. 


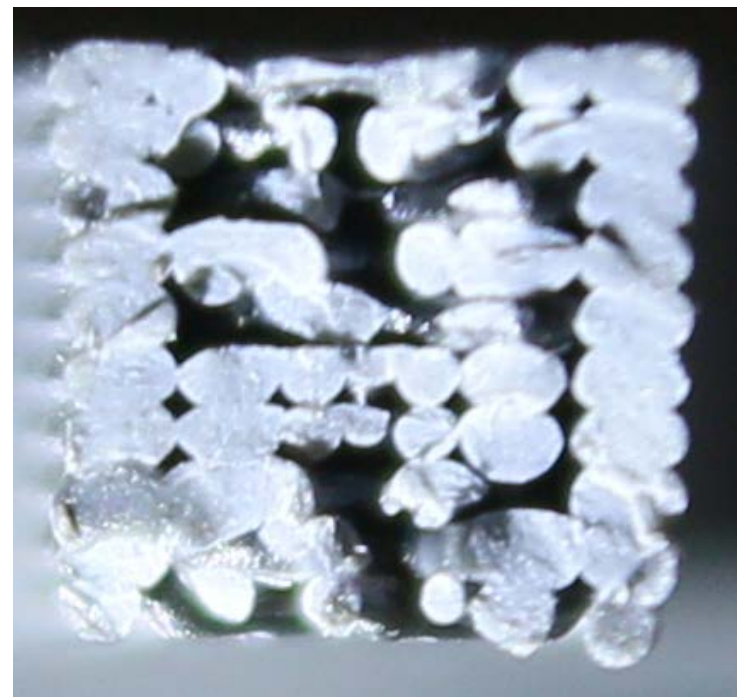

Fig. 10. HIPS fracture surface at $0.29 \mathrm{~mm}$ printed layer.

\section{Summary}

The presented study shows a case for the establishment of impact testing standards specifically for 3D-printed specimens. It is very important to have a deep knowledge about the mechanical behaviour of the 3D printed products, because nowadays the interest for 3D print is increasing in the plastics industry. The tensile impact testing of specimens printed from ABS and HIPS on a desktop FFF/FDM 3D printer yielded results which are statistically evaluated at each layer thickness. From this measurement implies that lower print layers are suitable for impact applications, but it all depends on time and overall economic demands.

This paper is supported by the internal grant of TBU in Zlin No. IGA/FT/2018/012 funded from the resources of specific university research, by the Czech Ministry of Industry and Trade in Program "Aplikace" No. CZ 01.1.02/0.0/0.0/16 084/0009949 and by the Ministry of Education, Youth and Sports of the Czech Republic within the National Sustainability Programme project No. LO1303 (MSMT-7778/2014) and also by the European Regional Development Fund under the project CEBIA-Tech No. CZ.1.05/2.1.00/03.0089.

\section{References}

1. D. Tomić, A. Fudurić, T. Mihalić, N. Simunić, JET 10 51-59 (2017)

2. H. Dodziuk, Kardiochir, Kardiologia Polska 13 283293 (2016)

3. D.H. Rosenzweig, E. Carelli, T. Steffen, P. Jarzem, L. Haglund, Int. J. Mol. Sci. 16 15118-15135 (2015)

4. V. Kovan, G. Altan, E.S. Topal, J. Mech. Sci. Technol. 31 2197-2201 (2017)

5. A. Gregor, E. Filova, J Kronek, H. Chlup, M. Buzgo, V. Blahnova, V. Lukasova, M. Bartos, A. Necas, J. Hosek, J. Biol. Eng. 11-31 (2017)
6. U. Ritz, R. Gerke, H. Götz, S. Stein, P.M. Rommens, Int. J. Mol. Sci. 18 (2017)

7. M. Vukicevic, B. Mosadegh, J.K. Min, S.H. Little, JACC: Cardiovasc. Imag. 10 171-184 (2017)

8. Ö. Keles, C.W. Blevins, K. Bowman, Rapid Prototyp. J. 23/2320-28 (2017)

9. J. Milde, L. Morovič, J. Blaha, MATEC Web. Conf. 137 (2017)

10. K. Szykiedans, W. Credo, D. Osinski, Procedia Eng. 177 455-461 (2017) 